Article

\title{
Entropy Principle and Galilean Relativity for Dense Gases, the General Solution without Approximations
}

\section{Maria Cristina Carrisi, Stefania Montisci and Sebastiano Pennisi *}

Dipartimento di Matematica ed Informatica, University of Cagliari, Via Ospedale 72, 09124 Cagliari, Italy; E-Mails: cristina.carrisi@tiscali.it (M.C.C.); montiscistefania@ tiscali.it (S.M.)

* Author to whom correspondence should be addressed; E-Mail: spennisi@unica.it; Tel.: +39-3280-092419; Fax: +39-0706-758501.

Received: 4 February 2013; in revised form: 23 February 2013 / Accepted: 27 February 2013 / Published: 11 March 2013

\begin{abstract}
The many moments model for dense gases and macromolecular fluids is considered here, where the upper order moment is chosen in accordance to the suggestions of the non-relativistic limit of the corresponding relativistic model. The solutions of the restrictions imposed by the entropy principle and that of Galilean relativity were, until now, obtained in the literature by using Taylor expansions around equilibrium and without proving convergence. Here, an exact solution without using expansions is found. The particular case with only 14 moments has already been treated in the literature in a completely different way. Here, it is proven that this particular closure is included in the presently more general one.
\end{abstract}

Keywords: extended thermodynamics; dense gas; Galilean relativity

Classification: PACS 80A17

\section{Introduction}

Extended Thermodynamics takes the first steps from the suggestions of kinetic theory of monatomic gases; here, the state of a gas is described by the phase density, $f(\vec{x}, \vec{c}, t)$, such that $f(\vec{x}, \vec{c}, t) d \vec{c}$ are the number density of atoms at the point, $\vec{x}$, and at time, $t$, that have velocities between $\vec{c}$ and $\vec{c}+d \vec{c}$. The phase density, also called the distribution function, obeys the Boltzmann equation:

$$
\partial_{t} f+c_{k} \partial_{k} f=q
$$


where external forces have been neglected and $q$ takes into account the effects of collisions between the atoms. After that, the moments are defined by:

$$
F^{i_{1} \cdots i_{n}}=\int f m c^{i_{1}} \cdots c^{i_{n}} d \vec{c}
$$

with $m$ the molecular mass. Multiplication of the Boltzmann equation by $m c^{i_{1}} \cdots c^{i_{n}}$ and integration over all $\vec{c}$ gives the balance equations for these moments. Some of these equations, and the corresponding moments, may also be considered only through their suitable traces. A corresponding definition can be formulated in the relativistic framework taking, after that, their non-relativistic limit. In this way, we obtain the following balance equations of the many moments model:

$$
\begin{aligned}
& \partial_{t} F^{i_{1} \cdots i_{n}}+\partial_{k} G^{k i_{1} \cdots i_{n}}=Q^{i_{1} \cdots i_{n}} \quad \text { for } n=0 \cdots, N \\
& \partial_{t} F_{R}^{i_{1} \cdots i_{R}}+\partial_{k} G_{R}^{k i_{1} \cdots i_{R}}=Q_{R}^{i_{1} \cdots i_{R}} \quad \text { for } R=0 \cdots, M
\end{aligned}
$$

where the infinite hierarchy of moments has been closed by choosing two arbitrary numbers, $N$ and $M$, such that $M<N$ and $M+N$ are odd numbers.

Other considerations [1,2] lead one to think that $N$ and $M$ are two subsequent numbers; this aspect does not affect the present work, so that we prefer to maintain their generality.

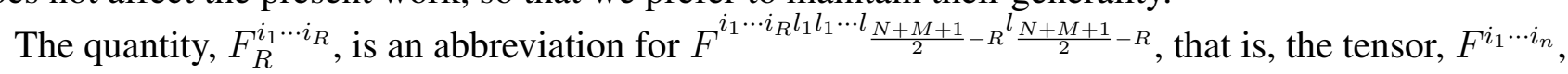
with $n=N+M+1-R$, of which we take the trace $\frac{N+M+1}{2}-R$-times.

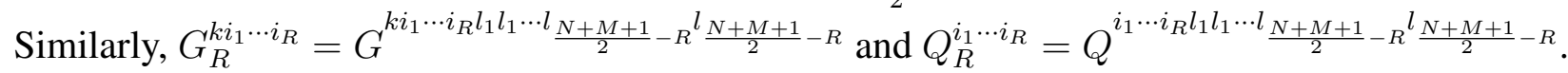

The moments, $F^{i_{1} \cdots i_{n}}$ and $F_{R}^{i_{1} \cdots i_{R}}$, can be taken as independent variables, while $G^{k i_{1} \cdots i_{n}}$ and $G_{R}^{k i_{1} \cdots i_{R}}$ are their fluxes and $Q^{i_{1} \cdots i_{n}}$ and $Q_{R}^{i_{1} \cdots i_{R}}$ are their production terms. All these quantities are symmetrical with respect to $i_{1} \cdots i_{n}$ and $i_{1} \cdots i_{R}$; in the particular case of monatomic gases, $G^{k i_{1} \cdots i_{n}}$ and $G_{R}^{k i_{1} \cdots i_{R}}$ are symmetric with respect to all their indexes, but for dense gases and macromolecular fluids, some evolutive terms appear also in the production terms; by taking them in the lefthand side of Equation (1), they may be included in the terms, $\partial_{k} G^{k i_{1} \cdots i_{n}}$ and $\partial_{k} G_{R}^{k i_{1} \cdots i_{R}}$, causing the loss of symmetry of $G^{k i_{1} \cdots i_{n}}$ and $G_{R}^{k i_{1} \cdots i_{R}}$ with respect to all their indexes. We remark that the kinetic theory of monatomic gases has been considered only to draw from it suggestions on the properties of moments and balance equations; it is assumed that these same properties hold also for dense gases and macromolecular fluids, except for the above mentioned symmetries.

Moreover, we have

- $Q=0, Q^{i}=0$ and $Q^{l l}=0$, so that Equation $(1)_{1}$ for $n=0, n=1$, and the trace of that for $n=2$ are the conservation laws of mass, momentum and energy, respectively. The other production terms, $Q^{i_{1} \cdots i_{n}}$ and $Q_{R}^{i_{1} \cdots i_{R}}$, are functions of the independent variables, which are zero at equilibrium; in the context of the present macroscopic approach, we may write the linear expressions of these functions with respect to equilibrium and find the sign of the coefficients by imposing that the density of entropy production is not negative; but we omit here these considerations for the sake of brevity.

- The following conditions can be considered:

$$
F^{k}=G^{k} \quad, \quad G^{k i}=G^{i k}
$$


The first one of these conditions surely holds and means that the flux in the conservation law of mass (i.e., Equation $(1)_{1}$ with $n=0$ ) is the independent variable in the conservation law of momentum (i.e., Equation $(1)_{1}$ for $n=1$ ).

The second condition, $(2)_{2}$, holds only if there is a conservation of angular momentum.

The balance Equation (1) becomes field equations when the fluxes and the productions are known functions of the independent variables. Restrictions on the generality of these functions are furnished by imposing the entropy principle and that of Galilean relativity.

In the particular case $N=2$ and $M=1$, we obtain the 14-moments model, which was firstly studied by Kremer [3,4] by imposing, up to the second order with respect to equilibrium, the conditions that come out from the above principles; subsequently, in [5,6], an exact non-approximated solution of these same conditions has been found. This result was achieved by writing firstly a relativistic model, for which it is easy to impose the Einstein relativity principle by using the representation theorems for isotropic functions, which are known with this number of variables [7-11], and then taking its non-relativistic limit.

An extension of this method, to the case with many moments, is not possible, because there are no known representation theorems for this general case.

In the present paper, we have found a different method to solve the above conditions. We describe these conditions in Section 2 and solve them in two different ways, which are reported in Section 3 and Section 4, respectively. Finally, in Section 5, we show that, in the particular case of the 14-moments model, they are equivalent to those of [5,6], even if these were found with a completely different method.

Obviously, all these considerations belong to the general context of Extended Thermodynamics, whose original and most important papers, in our opinion, are [12,13] and whose most important aspects are described in [14].

Some authors avoid considering Equation $(1)_{2}$ and, sometimes, also some components of Equation $(1)_{1}$ (see [15-21], for example); but, this is not physically acceptable, because it has been shown in $[22,23]$ that the form of Equation (1) is suggested by the non-relativistic limit of the relativistic model and that omitting Equation $(1)_{2}$ is equivalent to omitting the conservation law of mass or that of momentum energy in the corresponding relativistic model.

A similar result, restricted to the 14-moments model, was previously found in [24]. See also [6,25] for other details.

We conclude this section, remarking that this model has been obtained through a macroscopic approach, i.e., based on the entropy principle. Another possible formulation of Extended Thermodynamics for dense gases is based on the kinetic approach; in order to permit a comparison between the two approaches, we cite now some references on the kinetic approach. In [26], Enskog introduced a kinetic theory for dense gases, which yields a very good approximation of the behavior of gases. Later, hydrodynamic-like equations have been derived from the kinetic equation; see, for example, the Chapman-Enskog method [27].

In 1988, Kremer and Rosa [28] obtained hydrodynamic equations from the local equilibrium distribution function, as kernel linearizing the collision integral in Enskog's equation; in this way, they were able to derive sound dispersion relations for monatomic gases by using normal mode analysis. Based on this last paper, in 1991, Marques and Kremer [29] obtained linearized hydrodynamic equations 
involving the second order terms of the collision integral; in this way, they improved the results previously known in the literature and, furthermore, they obtained linearized Burnett equations for monatomic gases.

In [30], Ugawa and Cordero obtained extended hydrodynamic equations derived from Enskog's equation by using Grad's moment expansion method in the bi-dimensional case; among other results, they discussed the nature of a simple one-dimensional heat conduction problem and were able to show that, not too far from equilibrium, the non-equilibrium pressure in this case depends on the density, temperature and heat flux vector.

Finally, another model in this context can be found in [31], and this will surely be the object of further investigations in the future.

\section{The Entropy Principle and the Galilean Relativity Principle}

The entropy principle states that the supplementary law:

$$
\partial_{t} h+\partial_{k} h^{k}=\sigma \geq 0
$$

holds for every solution of Equation (1), where $h$ is the entropy density and $h^{k}$ its flux. For Liu's Theorem [32], this is equivalent to assuming the existence of Lagrange Multipliers, $\lambda_{i_{1} \cdots i_{n}}$ and $\lambda_{i_{1} \cdots i_{R}}^{R}$, such that:

$$
\begin{aligned}
& d h=\sum_{n=0}^{N} \lambda_{i_{1} \cdots i_{n}} d F^{i_{1} \cdots i_{n}}+\sum_{R=0}^{M} \lambda_{i_{1} \cdots i_{R}}^{R} d F_{R}^{i_{1} \cdots i_{R}} \\
& d h^{k}=\sum_{n=0}^{N} \lambda_{i_{1} \cdots i_{n}} d G^{k i_{1} \cdots i_{n}}+\sum_{R=0}^{M} \lambda_{i_{1} \cdots i_{R}}^{R} d G_{R}^{k i_{1} \cdots i_{R}}
\end{aligned}
$$

besides a residual inequality, which we leave out for the sake of brevity and because it does not affect the present results.

It is convenient to write Equation (4) in a more compact form by calling $F^{A}$ the variables, $F^{i_{1} \cdots i_{n}}$ and $F_{R}^{i_{1} \cdots i_{R}}$; in this way, Equation (4) can be written as:

$$
d h=\lambda_{A} d F^{A}, d h^{k}=\lambda_{A} d G^{k A}
$$

with the obvious meaning of $\lambda_{A}$ and $G^{k A}$.

In [33], the idea has been conceived of to define the four-potentials, $h^{\prime}$ and $h^{\prime k}$, as:

$$
h^{\prime}=-h+\lambda_{A} F^{A}, h^{\prime k}=-h^{k}+\lambda_{A} G^{k A}
$$

so that Equation (5) becomes:

$$
d h^{\prime}=F^{A} d \lambda_{A}, d h^{\prime k}=G^{k A} d \lambda_{A}
$$

Another idea exposed in [33] is to take the Lagrange Multipliers as independent variables and call them "main field"; in this way Equation (7) becomes:

$$
F^{A}=\frac{\partial h^{\prime}}{\partial \lambda_{A}} \quad, \quad G^{k A}=\frac{\partial h^{\prime k}}{\partial \lambda_{A}},
$$


so that everything is determined in terms of $h^{\prime}$ and $h^{\prime k}$; moreover, by substituting Equations (8) in (1), we obtain a symmetric system of evolution equations, which is hyperbolic if $h^{\prime}$ is a convex function of the main field. We observe here that it is not necessary to transform the system Equation (1) in the symmetric form, because if it is equivalent to a symmetric hyperbolic system, it itself is hyperbolic. Consequently, we can consider Equations $(5)_{1}$ and $(7)_{2}$ to still be maintaining the moments as independent variables. In this way, Equations $(5)_{1}$ and $(7)_{2}$ become:

$$
\lambda_{A}=\frac{\partial h}{\partial F^{A}} \quad, \quad \frac{\partial h^{\prime k}}{\partial F^{B}}=G^{k A} \frac{\partial^{2} h}{\partial F^{B} \partial F^{A}}
$$

which allows one to determine the unknown constitutive functions, $G^{k A}$, because the matrix, $\frac{\partial^{2} h}{\partial F^{B} \partial F^{A}}$, is an invertible matrix; otherwise, we could not invert Equation (9) ${ }_{1}$ and take the $\lambda_{A}$ as independent variables.

Let us introduce now the Galilean Relativity Principle.

To this end, it is firstly necessary to see how the moments and their fluxes transform under a change of frames moving, one with respect to the other, with a translational rectilinear uniform motion with velocity, $\vec{u}$. For the variables, $F^{i_{1} \cdots i_{n}}$ and $G^{k i_{1} \cdots i_{n}}$, it can be found in [34,35] and reads:

$$
\begin{aligned}
& F^{i_{1} \cdots i_{n}}=\sum_{s=0}^{n} S_{j_{1} \cdots j_{s}}^{i_{1} \cdots i_{n}}(\vec{u}) F^{I j_{1} \cdots j_{s}} \\
& G^{k i_{1} \cdots i_{n}}-\frac{F^{k}}{F} F^{i_{1} \cdots i_{n}}=\sum_{s=0}^{n} S_{j_{1} \cdots j_{s}}^{i_{1} \cdots i_{n}}(\vec{u})\left(G^{I k j_{1} \cdots j_{s}}-\frac{F^{I k}}{F^{I}} F^{I j_{1} \cdots j_{s}}\right)
\end{aligned}
$$

where $F^{I j_{1} \cdots j_{s}}$ and $G^{I k j_{1} \cdots j_{s}}$ are the counterparts of $F^{j_{1} \cdots j_{s}}$ and $G^{k j_{1} \cdots j_{s}}$ in the other reference frame; moreover:

$$
S_{j_{1} \cdots j_{s}}^{i_{1} \cdots i_{n}}(\vec{u})=\left(\begin{array}{c}
n \\
s
\end{array}\right) \delta_{j_{1}}^{\left(i_{1}\right.} \cdots \delta_{j_{s}}^{i_{s}} u^{i_{s+1}} \cdots u^{\left.i_{n}\right)}
$$

Regarding the other variables, we use the identity holding for $s \geq N+1$ :

$$
S_{j_{1} \cdots j_{s}}^{i_{1} \cdots i_{R} l_{1} l_{1} \cdots l_{\frac{N+M+1}{2}-R}} \frac{l_{\frac{N+M+1}{2}-R}}{2}=X_{\left(j_{1} \cdots j_{N+M+1-s}\right.}^{i_{1} \cdots i_{R}} \delta_{j_{N+M+2-s} j_{N+M+3-s}} \cdots \delta_{\left.j_{s-1} j_{s}\right)}
$$

with

$$
\begin{aligned}
& X_{j_{1} \cdots j_{N+M+1-s}}^{i_{1} \cdots i_{R}}=\sum_{(p, q) \in S}\left(\begin{array}{c}
R \\
s-q-2 p
\end{array}\right) 2^{q} \frac{\left(\frac{N+M+1}{2}-R\right) !}{p ! q !\left(\frac{N+M+1}{2}-R-p-q\right) !}\left(u^{2}\right)^{\frac{N+M+1}{2}-R-p-q} \\
& u_{\left(j_{1} \cdots u_{j_{q}} \delta_{j_{q+1} j_{q+2}} \cdots \delta_{j_{q+N+M+2 p-2 s} j_{q+N+M+2 p-2 s+1}} \delta_{j_{q+N+M+2 p-2 s+2}}^{\left(i_{1}\right.} \cdots \delta_{\left.j_{N+M+1-s}\right)}^{i_{s-q-2 p}}\right.} u^{i_{s-q-2 p+1}} \cdots u^{\left.i_{R}\right)}
\end{aligned}
$$

where $S$ is the set of the couples, $(p, q)$, of integer numbers, $p$ and $q$, such that $p \geq 0, q \geq 0$ and $p+q \leq \frac{N+M+1}{2}-R$ and $s-R \leq q+2 p \leq s$.

We omit here the proof of this identity, for the sake of brevity (see [36] ). Moreover, we define:

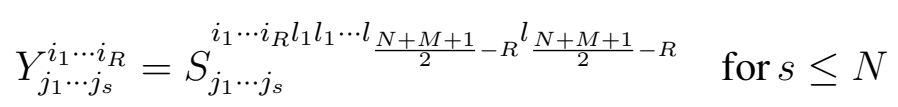


Now, we can extend Equation (10) also for the value $n=N+M+1-R$ and contract the result with $\delta_{i_{R+1} i_{R+2}} \cdots \delta_{i_{N+M-R} i_{N+M+1-R}}$; by using Equations (12) and (14) we obtain:

$$
\begin{aligned}
F_{R}^{i_{1} \cdots i_{R}} & =\sum_{s=0}^{N} Y_{j_{1} \cdots j_{s}}^{i_{1} \cdots i_{R}} F^{I j_{1} \cdots j_{s}}+\sum_{S=R}^{M} X_{j_{1} \cdots j_{S}}^{i_{1} \cdots i_{R}} F_{S}^{I j_{1} \cdots j_{S}} \\
G_{R}^{k i_{1} \cdots i_{R}}-\frac{F^{k}}{F} F_{R}^{i_{1} \cdots i_{R}}= & \sum_{s=0}^{N} Y_{j_{1} \cdots j_{s}}^{i_{1} \cdots i_{R}}\left(G^{I k j_{1} \cdots j_{s}}-\frac{F^{I k}}{F^{I}} F^{I j_{1} \cdots j_{s}}\right)+ \\
& +\sum_{S=R}^{M} X_{j_{1} \cdots j_{S}}^{i_{1} \cdots i_{R}}\left(G_{S}^{I k j_{1} \cdots j_{S}}-\frac{F^{I k}}{F^{I}} F_{S}^{I j_{1} \cdots j_{S}}\right)
\end{aligned}
$$

where, in the second summation, we have changed the index according to $S=N+M+1-s$.

Equations (10) and (15) can be written in a more compact form as:

$$
F^{A}=X_{B}^{A}(\vec{u}) F^{I B} \quad, \quad G^{k A}-\frac{F^{k}}{F} F^{A}=X_{B}^{A}(\vec{u})\left(G^{I k B}-\frac{F^{I k}}{F^{I}} F^{I B}\right)
$$

with the obvious meaning of $X^{A}{ }_{B}(\vec{u})$. This matrix satisfies the following properties:

$$
\begin{aligned}
& \text { (1) } X_{A}^{C}(-\vec{u}) X_{B}^{A}(\vec{u})=\delta_{B}^{C} \\
& \text { (2) } \frac{\partial X^{A}{ }_{C}(\vec{u})}{\partial u^{j}}=M^{j A}{ }_{B} X^{B}{ }_{C}(\vec{u})
\end{aligned}
$$

where $M^{j A}{ }_{B}$ is the constant matrix defined in the following way:

- If the multiindex $A$ is the same appearing in $F^{i_{1} \cdots i_{n}}$ with $n=0$, then $M^{j 0}{ }_{B}=0$;

- If the multiindex $A$ is the same appearing in $F^{i_{1} \cdots i_{n}}$ with $1 \leq n \leq N$, then

$$
M_{B}^{j i_{1} \cdots i_{n}}=\left\{\begin{array}{lll}
n \delta_{j_{1}}^{\left(i_{1}\right.} \cdots \delta_{j_{n-1}}^{i_{n-1}} \delta^{\left.i_{n}\right) j} & \text { if } & B=j_{1} \cdots j_{n-1} \\
0 & \text { if } \quad B \neq j_{1} \cdots j_{n-1}
\end{array}\right.
$$

- If the multiindex $A$ is the same appearing in $F_{R}^{i_{1} \cdots i_{R}}$ with $R=M$, then

$$
M_{B i_{1} \cdots i_{M}}^{j{ }_{B}}=\left\{\begin{array}{lll}
{\left[(N-M+1) \delta_{j_{M+1}}^{j} \delta_{j_{1}}^{\left(i_{1}\right.}+M \delta^{j\left(i_{1}\right.} \delta_{j_{M+1} j_{1}}\right] \delta_{j_{2}}^{i_{2}} \cdots \delta_{j_{M}}^{\left.i_{M}\right)} \cdot} & & \\
\cdot_{j_{M+2} j_{M+3}} \cdots \delta_{j_{N-1} j_{N}} & \text { if } & B=j_{1} \cdots j_{N} \\
0 & \text { if } & B \neq j_{1} \cdots j_{N}
\end{array}\right.
$$

- If the multiindex $A$ is the same appearing in $F_{R}^{i_{1} \cdots i_{R}}$ with $0 \leq R \leq M-1$, then

$$
M^{j i_{1} \cdots i_{R}}{ }_{B}=\left\{\begin{array}{lll}
{\left[(N+M+1-2 R) \delta_{j_{R+1}}^{j} \delta_{j_{1}}^{\left(i_{1}\right.}+R \delta^{j\left(i_{1}\right.} \delta_{j_{R+1} j_{1}}\right]} & \\
& \cdot \delta_{j_{2}}^{i_{2}} \cdots \delta_{j_{R}}^{\left.i_{R}\right)} & \text { if } B=j_{1} \cdots j_{R+1}, R \neq 0 \\
(N+M+1) \delta_{j_{1}}^{j} & \text { if } B=j_{1} \cdots j_{R+1}, R=0 \\
0 & \text { if } B \neq j_{1} \cdots j_{R+1}
\end{array}\right.
$$

Another property of the matrix, $X^{A}{ }_{B}$, is:

$$
\text { (3) } X_{B}^{A}(\vec{u}) M^{j B}{ }_{C}=M^{j A}{ }_{B} X^{B}{ }_{C}(\vec{u})
$$


The counterparts of these properties when only Equation $(1)_{1}$ is considered and Equation $(1)_{2}$ is omitted have been already found in [14]; now, we have found that they hold also for the complete system Equation (1), but we have reported their proofs in a separate paper [36], for the sake of brevity.

Now, we are ready to impose the Galilean relativity principle for our system. It can be subdivided in two parts:

- If we substitute Equation $(16)_{1}$ in $h$ and $h^{k}-h \frac{F^{k}}{F}$, we obtain composite functions; the principle states that they are non-convective quantities in the sense that they do not depend on $u^{j}$. In other words, they do not depend on the reference frame. To impose this restriction, we have simply to say that their derivatives with respect to $u^{j}$ are zero. By using Equations $(16)_{1},(18)$ and then, again, Equation $(16)_{1}$, we obtain:

$$
\frac{\partial h}{\partial F^{A}} M_{B}^{j A} F^{B}=0 \quad, \quad \frac{\partial h^{k}}{\partial F^{A}} M_{B}^{j A} F^{B}-h \delta^{k j}=0
$$

where we have used $M^{j}{ }_{B}=0$ and the subsequent property for $M^{j i_{1}}{ }_{B}$, reported after Equation (18).

We note that, as a consequence of Equation $(20)_{1}$, also $h^{\prime}$ is a non-convective quantity; in fact, from Equation $(9)_{1}$ and Equation $(6)_{1}$, it follows:

$h^{\prime}=-h+\frac{\partial h}{\partial F^{A}} F^{A}=-h+\frac{\partial h}{\partial F^{I B}} \frac{\partial F^{I B}}{\partial F^{A}} F^{A}=-h+\frac{\partial h}{\partial F^{I B}} X_{A}^{B}(-\vec{u}) F^{A}==-h+\frac{\partial h}{\partial F^{I B}} F^{I B}(21$

where, in the last two passages, we have used the inverse of Equation $(16)_{1}$, with the use of Equation (17).

- The second requirement imposed by the Galilean relativity principle is that the decomposition Equation $(16)_{2}$ holds for the fluxes.

As a consequence of this condition, it follows that also $h^{\prime k}-h^{\prime} \frac{F^{k}}{F}$ is a non-convective quantity. In fact, from Equation (6), we have:

$$
\begin{aligned}
h^{\prime k} & -h^{\prime} \frac{F^{k}}{F}=-\left(h^{k}-h \frac{F^{k}}{F}\right)+\lambda_{A}\left(G^{k A}-\frac{F^{k}}{F} F^{A}\right)= \\
& =-\left(h^{k}-h \frac{F^{k}}{F}\right)+\frac{\partial h}{\partial F^{A}}\left(G^{k A}-\frac{F^{k}}{F} F^{A}\right)= \\
& =-\left(h^{k}-h \frac{F^{k}}{F}\right)+\frac{\partial h}{\partial F^{I B}} X_{A}^{B}(-\vec{u})\left(G^{k A}-\frac{F^{k}}{F} F^{A}\right)= \\
& =-\left(h^{k}-h \frac{F^{k}}{F}\right)+\frac{\partial h}{\partial F^{I B}}\left(G^{I k B}-\frac{F^{I k}}{F^{I}} F^{I B}\right)
\end{aligned}
$$

where, in the second passage, we have used Equation $(9)_{1}$, in the third passage, we have used the inverse of Equation $(16)_{1}$ with the use of Equation (17) and, in the last passage, we have used the inverse of Equation $(16)_{2}$ with the use of Equation (17).

Consequently, Equation (20) has to be satisfied also with $h^{\prime}$ and $h^{\prime k}$ instead of $h$ and $h^{k}$, respectively, that is:

$$
\frac{\partial h^{\prime}}{\partial F^{A}} M_{B}^{j A} F^{B}=0 \quad, \quad \frac{\partial h^{\prime k}}{\partial F^{A}} M_{B}^{j A} F^{B}-h^{\prime} \delta^{k j}=0
$$


Vice versa, if we assume that $h$ and $h^{\prime k}-h^{\prime} \frac{F^{k}}{F}$ are non-convective quantities, then Equation $(16)_{2}$ will follow as a consequence and, also, the non-convectivity of $h^{k}-h \frac{F^{k}}{F}$. In fact, from Equation $(9)_{2}$, it follows:

$$
\frac{\partial h^{\prime k}}{\partial F^{I B}}=G^{k A} \frac{\partial^{2} h}{\partial F^{I B} \partial F^{A}}=G^{k A} \frac{\partial^{2} h}{\partial F^{I B} \partial F^{I C}} X_{A}^{C}(-\vec{u})
$$

where, in the last passage, we have used the inverse of Equation $(16)_{1}$. Similarly, always with the use of the inverse of Equation $(16)_{1}$, we obtain:

$$
\begin{aligned}
& -\frac{F^{k}}{F} F^{A} X_{A}^{C}(-\vec{u})=-\frac{F^{k}}{F} F^{I C} \quad \text { from which it follows } \\
& -\frac{F^{k}}{F} F^{A} X_{A}^{C}{ }_{A}(-\vec{u}) \frac{\partial^{2} h}{\partial F^{I B} \partial F^{I C}}=-\frac{F^{k}}{F} F^{I C} \frac{\partial^{2} h}{\partial F^{I B} \partial F^{I C}}
\end{aligned}
$$

Thanks to this equation and to Equation (24), we obtain:

$$
\left(G^{k A}-\frac{F^{k}}{F} F^{A}\right) X_{A}^{C}(-\vec{u}) \frac{\partial^{2} h}{\partial F^{I B} \partial F^{I C}}=\frac{\partial h^{\prime k}}{\partial F^{I B}}-\frac{F^{k}}{F} F^{I C} \frac{\partial^{2} h}{\partial F^{I B} \partial F^{I C}}
$$

But, from the first equality in Equation (21) written in the new reference frame, we have:

$$
\begin{aligned}
& \frac{\partial h}{\partial F^{I C}} F^{I C}=h^{\prime}+h, \quad \text { whose derivative with respect to } F^{I B} \text { is } \\
& \frac{\partial^{2} h}{\partial F^{I B} \partial F^{I C}} F^{I C}+\frac{\partial h}{\partial F^{I B}}=\frac{\partial h^{\prime}}{\partial F^{I B}}+\frac{\partial h}{\partial F^{I B}}
\end{aligned}
$$

This result allows one to rewrite Equation (25) as:

$$
\left(G^{k A}-\frac{F^{k}}{F} F^{A}\right) X_{A}^{C}(-\vec{u}) \frac{\partial^{2} h}{\partial F^{I B} \partial F^{I C}}=\frac{\partial h^{\prime k}}{\partial F^{I B}}-\frac{F^{k}}{F} \frac{\partial h^{\prime}}{\partial F^{I B}}
$$

Now, the hypothesis that $h^{\prime k}-h^{\prime} \frac{F^{k}}{F}$ is a non-convective quantity means that:

$$
h^{\prime k}-h^{\prime} \frac{F^{k}}{F}=h^{\prime I k}-h^{\prime} \frac{F^{I k}}{F^{I}}, \quad \text { from which } \quad h^{\prime k}=h^{\prime I k}+u^{k} h^{\prime}
$$

(Because $F=F^{I}, F^{k}=F^{I k}+F^{I} u^{k}$ ). By using this equation, we can write Equation (26) as:

$$
\left(G^{k A}-\frac{F^{k}}{F} F^{A}\right) X_{A}^{C}(-\vec{u}) \frac{\partial^{2} h}{\partial F^{I B} \partial F^{I C}}=\frac{\partial h^{I k}}{\partial F^{I B}}-\frac{F^{I k}}{F^{I}} \frac{\partial h^{\prime}}{\partial F^{I B}}
$$

Now, $\frac{\partial^{2} h}{\partial F^{I B} \partial F^{I C}}$ is a non-convective invertible matrix, so it follows that $\left(G^{k A}-\frac{F^{k}}{F} F^{A}\right) X^{C}{ }_{A}(-\vec{u})$ is a non-convective quantity, and this proves Equation $(16)_{2}$.

After that, we note that Equation (22) was deduced without using the non-convectivity of $h^{k}-h u^{k}$. In other words, we assumed that $h^{k}-h u^{k}$ is a non-convective quantity and proved with Equation (22) that also $h^{\prime k}-h^{\prime} u^{k}$ has this property; similarly, we assume now that $h^{\prime k}-h^{\prime} u^{k}$ is a non-convective quantity and prove through Equation (22) that also $h^{k}-h u^{k}$ satisfies this property.

We conclude that the Galilean relativity principle amounts simply in the conditions $(20)_{1}$ and $(23)_{2}$. 


\subsection{The Galilean Relativity Principle in Terms of the Main Field}

A more simple result holds when we take the Lagrange multipliers as independent variables; in fact, from Equations (5) and (16) $)_{1}$ it follows:

$$
d h=\lambda_{B}^{I} d F^{I B} \quad \text { with } \quad \lambda_{B}^{I}=X_{B}^{A}(\vec{u}) \lambda_{A}
$$

from which, by using Equation (17), it follows:

$$
\lambda_{C}=X_{C}^{B}(-\vec{u}) \lambda_{B}^{I}
$$

that is, the equation corresponding to Equation $(16)_{1}$, but for the Lagrange multipliers. ¿From Equation (29), it follows:

$$
\frac{\partial \lambda_{C}}{\partial u^{j}}=-M^{j E}{ }_{C} \lambda_{E}
$$

where Equations (18) and (19) and, again, Equation (29) have been used.

If we start now from the hypothesis that $h^{\prime}$ and $h^{\prime k}-h^{\prime} \frac{F^{k}}{F}$ are non-convective quantities, then the decomposition Equation $(16)_{1}$ follows as its consequence and of Equations $(24)_{2}$ and (8).

Similarly, with the use also of Equation (27), we have:

$$
\begin{gathered}
G^{k A}=\frac{\partial h^{\prime k}}{\partial \lambda_{B}^{I}} X^{A}{ }_{B}(\vec{u})=\left(\frac{\partial h^{\prime I k}}{\partial \lambda_{B}^{I}}+u^{k} \frac{\partial h^{\prime}}{\partial \lambda_{B}^{I}}\right) X_{B}^{A}(\vec{u}) \\
-\frac{F^{k}}{F} F^{A}=-\left(\frac{F^{I k}}{F^{I}}+u^{k}\right) F^{A}=-\left(\frac{F^{I k}}{F^{I}}+u^{k}\right) X_{B}^{A}(\vec{u}) F^{I B}= \\
=-\left(\frac{F^{I k}}{F^{I}} F^{I B}+u^{k} \frac{\partial h^{\prime}}{\partial \lambda_{B}^{I}}\right) X_{B}^{A}(\vec{u})
\end{gathered}
$$

The sum of these two equations gives Equation $(16)_{2}$.

Consequently, if we take the Lagrange multipliers as independent variables, the Galilean relativity principle amounts simply in imposing that $h^{\prime}$ and $h^{\prime k}-h^{\prime} \frac{F^{k}}{F}$ are non-convective quantities, that is. $\frac{\partial h^{\prime}}{\partial u^{j}}=0$ and $\frac{\partial h^{\prime I k}}{\partial u^{j}}=0$ with $h^{\prime I k}$ deduced from Equation (27).

In other words, it is expressed by:

$$
\lambda_{D} M_{A}^{j D} \frac{\partial h^{\prime}}{\partial \lambda_{A}}=0 \quad, \quad \lambda_{D} M^{j D} \frac{\partial h^{\prime k}}{\partial \lambda_{A}}+h^{\prime} \delta^{k j}=0
$$

where we have used Equation (30).

In the next section, we will find the general solution of Equations $(20)_{1}$ and $(23)_{2}$ in the independent variables $F^{A}$, while in Section 4, we will find the general solution of Equation (31) in the independent variables $\lambda_{A}$.

Before ending this section, we note that in the earlier papers on Extended Thermodynamics, the independent variables, $v^{i}$ and $m^{A}$, were used, which are defined by Equation $(16)_{1}$ with $v^{i}$ instead of $u^{i}$, $F^{I i}=0$ and $F^{I B}=m^{B}$ for $B \neq i$.

Similarly, the dependent variables, $m^{k A}$, were defined by Equation $(16)_{2}$ with $v^{i}$ instead of $u^{i}$ and $F^{I k}=0$ and $m^{k B}$ instead of $G^{I k B}$.

If we want to express the present results in terms of these other variables, we note that 
- Equations $(8)_{1}$ with $F^{A}=X^{A}{ }_{B}(\vec{v}) m^{B}$ are still a system of implicit equations for the determination of the Lagrange multipliers in terms of $v^{i}$ (velocity) and $m^{A}$. After that, Equation (31) means that the composite functions, $h^{\prime}$ and $h^{\prime k}-v^{k} h^{\prime}$, do not depend on $v^{i}$.

- By substituting $\lambda_{A}=\lambda_{A}\left(v^{i}, m^{B}\right)$ into Equation $(8)_{2}$, the resulting function, $G^{k A}$, satisfy the relation, $G^{k A}-\frac{F^{k}}{F} F^{A}=X_{B}^{A}(\vec{v}) m^{k B}$. In fact, we have:

$$
\begin{aligned}
& \frac{\partial}{\partial v^{j}}\left[X_{A}^{C}(-\vec{v})\left(G^{k A}-v^{k} F^{A}\right)\right] \stackrel{1}{=}-M_{B}^{j C} X_{B}^{B}{ }_{A}(-\vec{v})\left(G^{k A}-v^{k} F^{A}\right)+ \\
& +X^{C}{ }_{A}(-\vec{v})\left[-\delta^{k j} F^{A}+\left(\frac{\partial G^{k A}}{\partial \lambda_{B}}-v^{k} \frac{\partial F^{A}}{\partial \lambda_{B}}\right) \frac{\partial \lambda_{B}}{\partial v^{j}}\right] \stackrel{2}{=} \\
& =-M^{j C}{ }_{B} X^{B}{ }_{A}(-\vec{v})\left(\frac{\partial h^{\prime k}}{\partial \lambda_{A}}-v^{k} \frac{\partial h^{\prime}}{\partial \lambda_{A}}\right)+X_{A}^{C}(-\vec{v})\left[-\delta^{k j} \frac{\partial h^{\prime}}{\partial \lambda_{A}}+\right. \\
& \left.+\left(\frac{\partial^{2} h^{\prime k}}{\partial \lambda_{A} \partial \lambda_{B}}-v^{k} \frac{\partial^{2} h^{\prime}}{\partial \lambda_{A} \partial \lambda_{B}}\right) \frac{\partial \lambda_{B}}{\partial v^{j}}\right] \underline{\underline{3}} \\
& =-X_{B}^{C}(-\vec{v}) M^{j B}{ }_{A}\left(\frac{\partial h^{\prime k}}{\partial \lambda_{A}}-v^{k} \frac{\partial h^{\prime}}{\partial \lambda_{A}}\right)+X_{A}^{C}(-\vec{v})\left[-\delta^{k j} \frac{\partial h^{\prime}}{\partial \lambda_{A}}+\right. \\
& \left.-\left(\frac{\partial^{2} h^{\prime k}}{\partial \lambda_{A} \partial \lambda_{B}}-v^{k} \frac{\partial^{2} h^{\prime}}{\partial \lambda_{A} \partial \lambda_{B}}\right) M^{j E}{ }_{B} \lambda_{E}\right]= \\
& =-X^{C}{ }_{A}(-\vec{v})\left[M^{j A}{ }_{E}\left(\frac{\partial h^{\prime k}}{\partial \lambda_{E}}-v^{k} \frac{\partial h^{\prime}}{\partial \lambda_{E}}\right)+\right. \\
& \left.+\delta^{k j} \frac{\partial h^{\prime}}{\partial \lambda_{A}}+\left(\frac{\partial^{2} h^{\prime k}}{\partial \lambda_{A} \partial \lambda_{B}}-v^{k} \frac{\partial^{2} h^{\prime}}{\partial \lambda_{A} \partial \lambda_{B}}\right) M_{B}^{j E}{ }_{B}\right] \underline{4}=0
\end{aligned}
$$

where, in the passage denoted with $\stackrel{1}{=}$, we have used Equation (18), in the passage denoted with $\stackrel{2}{=}$, we have used Equation (8) and in the passage denoted with $\stackrel{3}{=}$, we have used Equation (19) and also Equation (30); this last one can be used, because it was deduced, taking into account only the fact that Equation (28) and Equation (29) are invertible relations between $\lambda_{A}$ and $\lambda_{B}^{I}$, without worrying if these are independent variables or not.

Finally, in the passage denoted with $\stackrel{4}{=}$, we have taken into account the fact that the coefficient of $-v^{k}$ inside the square brackets is Equation $(31)_{1}$ written with $E$ instead of $A$ and, after that, derivated with respect to $\lambda_{A}$; the remaining part is the derivative with respect to $\lambda_{A}$ of Equation $(31)_{2}$, written with $E$ instead of $A$ and, after that, derived with respect to $\lambda_{A}$.

So, we have proven that the usual decomposition in terms of $v^{i}, m^{A} \neq m^{i}$ and $m^{k A}$ is a consequence of our Equation (31). In this way, the only unknowns are the functions, $h^{\prime}, h^{\prime k}-h^{\prime} v^{k}$ and $m^{k A}$, depending on the variables, $m^{A} \neq m^{i}$.

To find these unknowns, we can use Equation (8) to obtain $\lambda_{A}$ as functions of $F^{B}$, substitute them in $h^{\prime}, h^{\prime k}-h^{\prime} v^{k}$ and $G^{k A}$ and, after that, calculate this in $v^{i}=0$, so obtaining $h^{\prime}, h^{\prime k}-h^{\prime} v^{k}$ and $m^{k A}$. However, this is equivalent to calculate Equation (8) in $v^{i}=0$, so that they become:

$$
m^{A}=\frac{\partial h^{\prime}}{\partial \lambda_{A}} \text { for } A \neq i \quad, \quad 0=\frac{\partial h^{\prime}}{\partial \lambda_{i}} \quad, \quad m^{k A}=\frac{\partial h^{\prime k}}{\partial \lambda_{A}}
$$

After that, we use Equation (32) $)_{1,2}$ to obtain $\lambda_{A}$ and $\lambda_{i}$ in terms of $m^{A} \neq m^{i}$ and substitute them in Equation (32) $)_{3}$ and in the expressions of $h^{\prime}$ and $h^{\prime k}$, calculated in $v^{i}=0$. This is the essence of the method explained in $[37,38]$ and which was already been used in $[39,40]$. 


\section{The General Solution in Terms of the Moments, $F^{A}$, as Independent Variables}

We find now the general solution of Equations $(20)_{1}$ and $(23)_{2}$ in the independent variables, the moments, $F^{A}$. Let us begin with Equation $(20)_{1}$.

To this end, let us call $F^{A^{*}}$ the variables, $F^{A}$, different from $F$ and $F^{j}$. With this notation, Equation $(20)_{1}$ becomes:

$$
\frac{\partial h}{\partial F^{j}} F+\frac{\partial h}{\partial F^{A^{*}}} M^{j A^{*}} F^{B}=0
$$

It is not restrictive to consider $h$ a composite function of:

$$
\begin{aligned}
& H\left(F, F^{i}, \eta^{B^{*}}\right) \text { and of } \eta^{B^{*}}=X_{A}^{B^{*}}\left(-\frac{F^{i}}{F}\right) F^{A}, \quad \text { that is } \\
& h=H\left[F, F^{i}, X_{A}^{A^{*}}\left(-\frac{F^{i}}{F}\right) F^{A}\right]
\end{aligned}
$$

By using this expression and Equation (18), the Equation (33) becomes:

$$
\begin{gathered}
F\left\{\frac{\partial H}{\partial F^{j}}+\frac{\partial H}{\partial \eta^{B^{*}}}\left[-\frac{1}{F} M^{j B^{*}}{ }_{D} X^{D}{ }_{A}\left(-\frac{F^{i}}{F}\right) F^{A}+X^{B^{*}}\left(-\frac{F^{i}}{F}\right)\right]\right\}+ \\
+\frac{\partial H}{\partial \eta^{B^{*}}} X^{B^{*}}{ }_{A^{*}}\left(-\frac{F^{i}}{F}\right) M^{j A^{*}}{ }_{B} F^{B}=0
\end{gathered}
$$

However, for Equation (19), the first term in the square brackets of Equation (35) becomes:

$$
\begin{gathered}
-\frac{1}{F} M^{j B^{*}}{ }_{D} X^{D}{ }_{A}\left(-\frac{F^{i}}{F}\right) F^{A}=-\frac{1}{F} X^{B^{*}}{ }_{D}\left(-\frac{F^{i}}{F}\right) M^{j D}{ }_{A} F^{A}= \\
=-\frac{1}{F}\left[X^{B^{*}}{ }_{0}\left(-\frac{F^{i}}{F}\right) M^{j 0}{ }_{A} F^{A}+X^{B^{*}}{ }_{i_{1}}\left(-\frac{F^{i}}{F}\right) M^{j i_{1}}{ }_{A} F^{A}+\right. \\
\left.+X^{B^{*}}{ }_{D^{*}}\left(-\frac{F^{i}}{F}\right) M^{j D^{*}}{ }_{A} F^{A}\right]
\end{gathered}
$$

This allows one to rewrite Equation (35) as:

$$
F \frac{\partial H}{\partial F^{j}}+\frac{\partial H}{\partial \eta^{B^{*}}}\left[-X^{B^{*}}{ }_{0}\left(-\frac{F^{i}}{F}\right) M^{j 0}{ }_{A} F^{A}-X^{B^{*}}{ }_{i 1}\left(-\frac{F^{i}}{F}\right) M^{j i_{1}}{ }_{A} F^{A}+F X^{B^{*}}{ }_{j}\left(-\frac{F^{i}}{F}\right)\right]=0
$$

By using the expression of $M^{j A}{ }_{B}$ reported after Equation (18), our condition becomes $\frac{\partial H}{\partial F^{j}}=0$, that is, $H$ does not depend on $F^{j}$. In other words, the general solution of Equation $(20)_{1}$ is:

$$
h=H\left[F, X_{A}^{A^{*}}\left(-\frac{F^{i}}{F}\right) F^{A}\right] .
$$

Let us find now the general solution of Equation $(23)_{2}$.

To this end, it will be useful to know firstly the expression of $h^{\prime}$ deduced from the first equality in Equation (21) and from Equation (37), that is:

$$
h^{\prime}=-h+\frac{\partial h}{\partial F} F+\frac{\partial h}{\partial F^{j}} F^{j}+\frac{\partial h}{\partial F^{B^{*}}} F^{B^{*}}=-H\left[F, X_{A}^{A^{*}}\left(-\frac{F^{i}}{F}\right) F^{A}\right]+
$$




$$
\begin{aligned}
& +F\left\{\frac{\partial H}{\partial F}+\frac{\partial H}{\partial \eta^{A^{*}}} M^{j A^{*}}{ }_{B} X^{B}{ }_{A}\left(-\frac{F^{i}}{F}\right) F^{A} \frac{1}{F^{2}} F^{j}+\frac{\partial H}{\partial \eta^{A^{*}}} X^{A^{*}}\left(-\frac{F^{i}}{F}\right)\right\}+ \\
& +F^{j}\left\{-\frac{1}{F} \frac{\partial H}{\partial \eta^{A^{*}}} M^{j A^{*}}{ }_{B} X^{B}{ }_{A}\left(-\frac{F^{i}}{F}\right) F^{A}+\frac{\partial H}{\left.\partial \eta^{A^{*}} X^{A^{*}}\left(-\frac{F^{i}}{F}\right)\right\}+}\right. \\
& +F^{B^{*}} \frac{\partial H}{\partial \eta^{A^{*}}} X^{A^{*}}{ }_{B^{*}}\left(-\frac{F^{i}}{F}\right)
\end{aligned}
$$

where we have used Equation (18). Moreover, from Equation $(34)_{2}$, we see that the underlined terms contribute with $\frac{\partial H}{\partial \eta^{A^{*}}} \eta^{A^{*}}$. Two other terms in Equation (38) are opposite, and there remains:

$$
h^{\prime}=-H+F \frac{\partial H}{\partial F}+\frac{\partial H}{\partial \eta^{A^{*}}} \eta^{A^{*}}
$$

In other words, also $h^{\prime}$ has an expression like Equation (37), and this is not strange, because we have already said that Equation $(23)_{1}$ holds as a consequence of Equation $(20)_{1}$; moreover, Equation $(23)_{1}$ is the same Equation $(20)_{1}$, but with $h^{\prime}$ instead of $h$.

Let us find now the general solution of Equation $(23)_{2}$, that is $\frac{\partial h^{\prime k}}{\partial F^{j}} F+\frac{\partial h^{\prime k}}{\partial F^{A^{*}}} M^{j A^{*}}{ }_{B} F^{B}-h^{\prime} \delta^{k j}=0$; It is not restrictive to write

$$
h^{\prime k}=h^{\prime} \frac{F^{k}}{F}+H^{\prime k}\left[F, F^{i}, X_{A}^{A^{*}}\left(-\frac{F^{i}}{F}\right) F^{A}\right]
$$

which allows one to rewrite our equation as:

$$
\begin{gathered}
F\left\{\frac{h^{\prime}}{F} \delta^{k j}+\frac{\partial H^{\prime k}}{\partial F^{j}}+\frac{\partial H^{\prime k}}{\partial \eta^{B^{*}}}\left[-\frac{1}{F} M^{j B^{*}}{ }_{D} X^{D}{ }_{A}\left(-\frac{F^{i}}{F}\right) F^{A}+X^{B^{*}}{ }_{j}\left(-\frac{F^{i}}{F}\right)\right]\right\}+ \\
+\frac{\partial H^{\prime k}}{\partial \eta^{B^{*}}} X^{B^{*}} A^{*}\left(-\frac{F^{i}}{F}\right) M^{j A^{*}}{ }_{B} F^{B}-h^{\prime} \delta^{k j}=0
\end{gathered}
$$

where we have taken into account that $h^{\prime}$ satisfies Equation (23) . By using Equation (36), there remains

$$
F \frac{\partial H^{\prime k}}{\partial F^{j}}+\frac{\partial H^{\prime k}}{\partial \eta^{B^{*}}}\left[-X_{0}^{B^{*}}\left(-\frac{F^{i}}{F}\right) M^{j 0}{ }_{A} F^{A}-X_{i_{1}}^{B^{*}}\left(-\frac{F^{i}}{F}\right) M^{j i_{1}}{ }_{A} F^{A}+F X^{B^{*}}{ }_{j}\left(-\frac{F^{i}}{F}\right)\right]=0
$$

which, by using the expression of $M^{j A}{ }_{B}$ reported after Equation (18), becomes $\frac{\partial H^{\prime k}}{\partial F^{j}}=0$, that is, $H^{\prime k}$ does not depend on $F^{j}$. In other words, the general solution of Equation $(23)_{2}$ is:

$$
h^{\prime k}=h^{\prime} \frac{F^{k}}{F}+H^{\prime k}\left[F, X_{A}^{A^{*}}\left(-\frac{F^{i}}{F}\right) F^{A}\right]
$$

We note now that Equation (20) looks like Equation (23), but with $\left(h, h^{k}\right)$ instead of $\left(h^{\prime}, h^{\prime k}\right)$; this allows one to write the most general solution of Equation $(20)_{2}$ like Equation (40), that is,

$$
h^{k}=h \frac{F^{k}}{F}+H^{k}\left[F, X_{A}^{A^{*}}\left(-\frac{F^{i}}{F}\right) F^{A}\right]
$$

It is interesting to test our general solution in the particular case, $N=2$ and $M=1$, that is, the 14-moments case, where Equations $(20)_{1}$ and $(23)_{2}$ are:

$$
\begin{aligned}
& F \frac{\partial h}{\partial F^{j}}+2 F^{i} \frac{\partial h}{\partial F^{j i}}+\left(2 F^{i j}+F^{l l} \delta^{i j}\right) \frac{\partial h}{\partial F^{i l l}}+4 F^{j l l} \frac{\partial h}{\partial F^{a a b b}}=0 \\
& F_{\frac{\partial h^{\prime k}}{\partial F^{j}}}+2 F^{i} \frac{\partial h^{\prime k}}{\partial F^{j i}}+\left(2 F^{i j}+F^{l l} \delta^{i j}\right) \frac{\partial h^{\prime k}}{\partial F^{i l l}}+4 F^{j l l} \frac{\partial h^{\prime k}}{\partial F^{a a b b}}-h^{\prime} \delta k j=0
\end{aligned}
$$


In this case, the variable Equation $(34)_{2}$ is:

$$
\begin{aligned}
& \eta^{i j}=F^{i j}-\frac{1}{F} F^{i} F^{j} \\
& \eta^{i l l}=F^{i l l}-\frac{2}{F} F^{i l} F_{l}-\frac{1}{F} F^{l l} F^{i}+\frac{2}{F^{2}} F^{i} F^{l} F_{l} \\
& \eta^{a a b b}=F^{a a b b}-\frac{4}{F} F^{a a b} F_{b}+\frac{2}{F^{2}} F^{l l} F^{a} F_{a}+\frac{4}{F^{2}} F^{a b} F_{a} F_{b}-\frac{3}{F^{3}}\left(F^{a} F_{a}\right)^{2}
\end{aligned}
$$

and it is easy to verify that,

$$
\begin{aligned}
& h=H\left(F, \eta^{i j}, \eta^{i l l}, \eta^{a a b b}\right) \\
& h^{\prime k}=\frac{F^{k}}{F} H^{\prime}\left(F, \eta^{i j}, \eta^{i l l}, \eta^{a a b b}\right)+H^{\prime k}\left(F, \eta^{i j}, \eta^{i l l}, \eta^{a a b b}\right)
\end{aligned}
$$

in composite with Equation (43), are solutions of Equation (42).

\section{The General Solution in Terms of the Lagrange Multipliers as Independent Variables}

Let us now describe the general solution of Equation (31), using $\lambda_{A}$ as independent variables. It holds only in the subdomain with $\lambda^{0} \neq 0$, like Equations $(20)_{1}$ and $(23)_{2}$ were solved in the previous section, in the subdomain $F \neq 0$. However, $F$ is the mass density, so that it is obvious that it is different from zero. The same thing cannot be said for $\lambda^{0}$, which is zero, quite in equilibrium!

However, if we assume that $\lambda_{i}^{1}$ in equilibrium is an infinitesimal of a higher order with respect to $\lambda^{0}$, then our solution will be acceptable also at equilibrium. This assumption has been already adopted in $[6,25]$ for the 14-moments model and was based on the requirement of the convexity of entropy, which, in turns, assures the hyperbolicity of the resulting system.

Let us call $\lambda_{\tilde{A}}$ the variables $\lambda_{A}$, different from $\lambda^{0}$ and $\lambda_{i}^{1}$. With this notation, Equation (31) becomes:

$$
\lambda_{D} M_{\tilde{A}}^{j D} \frac{\partial h^{\prime}}{\partial \lambda_{\tilde{A}}}+\lambda_{D} M^{j D}{ }_{i} \frac{\partial h^{\prime}}{\partial \lambda_{i}^{1}}=0 \quad, \quad \lambda_{D} M_{\tilde{A}}^{j D} \frac{\partial h^{\prime k}}{\partial \lambda_{\tilde{A}}}+\lambda_{D} M^{j D_{i}} \frac{\partial h^{\prime k}}{\partial \lambda_{i}^{1}}+h^{\prime} \delta^{k j}=0
$$

where we have taken into account that $M^{j D}{ }_{A}=0$ when $A$ is the same index of $\lambda_{j_{1} \cdots j_{R}}^{R}$ and $R=0$; this fact is evident from the description of $M^{j D}{ }_{A}$, reported above, after Equation (18).

It is now not restrictive to consider $h^{\prime}$ a composite function of

$$
H^{\prime}\left(\mu_{\tilde{A}}, \lambda_{i}^{1}, \lambda^{0}\right) \quad \text { and of } \quad \mu_{\tilde{B}}=\lambda_{A} X_{\tilde{B}}^{A}\left(-\frac{1}{N+M+1} \frac{\lambda_{i}^{1}}{\lambda^{0}}\right)
$$

In this way, Equation $(45)_{1}$, by using Equation (18), becomes:

$$
\begin{aligned}
& \lambda_{D} M^{j D}{ }_{\tilde{A}} \frac{\partial H^{\prime}}{\partial \mu_{\tilde{B}}} X_{\tilde{B}}^{\tilde{A}}\left(-\frac{1}{N+M+1} \frac{\lambda_{i}^{1}}{\lambda^{0}}\right)+\lambda_{D} M^{j D}{ }_{i l_{1} l_{1} \cdots l_{\frac{N+M-1}{2}}} l_{\frac{N+M-1}{2}}\left\{\frac{\partial H^{\prime}}{\partial \lambda_{i}^{1}}+\right. \\
& \left.+\frac{\partial H^{\prime}}{\partial \mu_{\tilde{B}}}\left[X_{\tilde{B}}^{i l_{1} l_{1} \cdots l_{\frac{N+M-1}{2}}} \frac{l^{\frac{N+M-1}{2}}}{2}+\lambda_{A} M^{i A}{ }_{C} X^{C}{ }_{\tilde{B}}\left(-\frac{1}{N+M+1} \frac{\lambda_{i}^{1}}{\lambda^{0}}\right) \cdot \frac{-1}{N+M+1} \frac{1}{\lambda^{0}}\right]\right\}=0
\end{aligned}
$$

where we have substituted $M^{j D}{ }_{i}$ with $M^{j D}{ }_{i l_{1} l_{1} \cdots l} \frac{N+M-1}{2} l_{\frac{N+M-1}{2}}$ in order not to lose sight the fact that $i$ is the same index of $\lambda_{i}^{1}$.

Now, from the description of $M^{j D}$ A reported above, after Equation (18), we have:

$$
\begin{aligned}
& M^{j A}{ }_{l_{1} l_{1} \cdots l_{\frac{N+M+1}{2}}} l_{\frac{N+M+1}{2}}=0, M^{j D}{ }_{i l_{1} l_{1} \cdots l_{\frac{N+M-1}{2}}} l_{\frac{N+M-1}{2}}=0 \text { for } D \neq l_{1} l_{1} \cdots l_{\frac{N+M+1}{2}} l_{\frac{N+M+1}{2}}, \\
& M^{j l_{1} l_{1} \cdots l_{\frac{N+M+1}{2}}} \frac{l_{N+M+1}}{2}{ }_{i l} l_{1} \cdots l_{\frac{N+M-1}{2}} l_{\frac{N+M-1}{2}}=(N+M+1) \delta_{i}^{j}
\end{aligned}
$$


By using these equations, the relation, Equation (47), becomes:

$$
\begin{aligned}
& \lambda_{D} M_{\tilde{A}}^{j D} \frac{\partial H^{\prime}}{\partial \mu_{\tilde{B}}} X_{\tilde{B}}^{\tilde{A}}+\lambda^{0}(N+M+1) \frac{\partial H^{\prime}}{\partial \lambda_{j}^{1}}+
\end{aligned}
$$

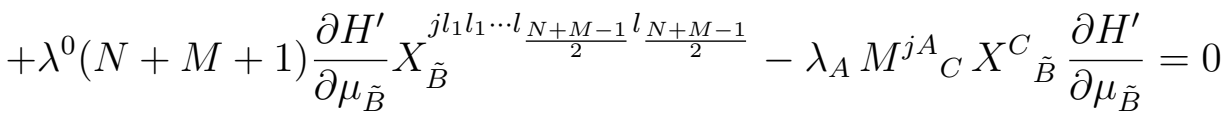

Now, we see that, from the first and last term, in this equation, there remains:

$$
\begin{gathered}
-\lambda_{A} M_{i_{1} l_{1} l_{1} \cdots l_{\frac{N+M-1}{2}} l_{\frac{N+M-1}{2}}} X_{\tilde{B}}^{i_{1} l_{1} l_{1} \cdots l_{\frac{N+M-1}{2}}} \frac{l_{N+M-1}}{2} \frac{\partial H^{\prime}}{\partial \mu_{\tilde{B}}}= \\
=-\lambda^{0}(N+M+1) X_{\tilde{B}}^{j l_{1} l_{1} \cdots l_{\frac{N+M-1}{2}}} \frac{l_{N+M-1}}{2} \frac{\partial H^{\prime}}{\partial \mu_{\tilde{B}}}
\end{gathered}
$$

where we have used Equation $(48)_{2,3}$. It is now evident that from Equation (49), it remains $\lambda^{0}(N+M+1) \frac{\partial H^{\prime}}{\partial \lambda_{j}^{1}}=0$, that is, $H^{\prime}$ does not depend on $\lambda_{j}^{1}$. In other words, the general solution of Equation $(31)_{1}$ is:

$$
h^{\prime}=H^{\prime}\left[\lambda_{A} X_{\tilde{B}}^{A}\left(-\frac{1}{N+M+1} \frac{\lambda_{i}^{1}}{\lambda^{0}}\right), \lambda^{0}\right]
$$

It remains now to find the general solution of Equation $(31)_{2}$. By defining $H^{\prime k}$ from $h^{\prime k}=H^{\prime k}-\frac{1}{N+M+1} \frac{\lambda_{k}^{1}}{\lambda^{0}} h^{\prime}$, it becomes:

$$
\lambda_{D} M_{A}^{j D} \frac{\partial H^{\prime k}}{\partial \lambda_{A}}=0
$$

where we have used the fact that $h^{\prime}$ already satisfies Equation $(31)_{1}$ and taken into account Equation (48). Now, Equation (51) looks like Equation $(31)_{1}$, but with $H^{\prime k}$ instead of $h^{\prime}$; by operating in the same way, we obtain that $H^{\prime k}$ is a composite function of $\lambda^{0}$ and $\lambda_{A} X^{A}{ }_{\tilde{B}}\left(-\frac{1}{N+M+1} \frac{\lambda_{i}^{1}}{\lambda^{0}}\right)$. In other words, the general solution of Equation $(31)_{2}$ is:

$$
h^{\prime k}=-\frac{1}{N+M+1} \frac{\lambda_{k}^{1}}{\lambda^{0}} h^{\prime}+H^{\prime k}\left[\lambda_{A} X_{\tilde{B}}^{A}\left(-\frac{1}{N+M+1} \frac{\lambda_{i}^{1}}{\lambda^{0}}\right), \lambda^{0}\right]
$$

Let us now see how the conditions (2) can be solved in terms of the functions, $H^{\prime}$ and $H^{\prime k}$, appearing in Equations (50) and (52), respectively.

\subsection{On the Condition $G^{k}=F^{k}$}

Let us consider now the condition $(2)_{1}$, which means that the flux in the conservation law of mass is the momentum density, that is, the independent variable in the subsequent conservation law of momentum. By using Equation (8), it can be expressed as:

$$
\frac{\partial h^{\prime k}}{\partial \lambda}=\frac{\partial h^{\prime}}{\partial \lambda^{k}}
$$

However, we note that $\lambda$ appears in $\mu_{\tilde{B}}$, as expressed by Equation $(46)_{2}$, only for $\tilde{B}=0$, that is, in $\tilde{\mu}$; moreover, we have:

$$
\frac{\partial \tilde{\mu}}{\partial \lambda}=1
$$


Similarly, $\lambda_{i}$ appears in $\mu_{\tilde{B}}$ only in $\tilde{\mu}$ and $\tilde{\mu}_{j}$; moreover, we have:

$$
\frac{\partial \tilde{\mu}}{\partial \lambda_{i}}=-\frac{1}{N+M+1} \frac{\lambda_{i}^{1}}{\lambda^{0}}, \quad \frac{\partial \tilde{\mu}_{j}}{\partial \lambda}=0, \quad \frac{\partial \tilde{\mu}_{j}}{\partial \lambda_{i}}=\delta i j
$$

In order to prove these properties, we note that from Equation $(16)_{1}$, it follows $\lambda_{A} F^{A}=\lambda_{A} X^{A}{ }_{B}(\vec{u}) F^{I B}$; by comparing this with the definition $(46)_{2}$ of $\mu_{\tilde{B}}$, we find that $\tilde{\mu}$ is the coefficient of $F^{I}$ in $\lambda_{A} F^{A}$, calculated in $u_{i}=-\frac{1}{N+M+1} \frac{\lambda_{i}^{1}}{\lambda^{0}}$ and $\tilde{\mu}_{j}$ is the coefficient of $F^{I j}$ in $\lambda_{A} F^{A}$, calculated in the previous value of $u_{i}$ (in effect, this value of $u^{i}$ was introduced above when we considered $h^{\prime}$ a composite function of Equation (46). This was inspired by the fact that, from Equation $(28)_{2}$ with $B=i l_{1} l_{1} \cdots l_{\frac{N+M-1}{2}} l_{\frac{N+M-1}{2}}$, this value $u_{i}$ corresponds to the condition $\lambda_{i l_{1} l_{1} \cdots l_{\frac{N+M-1}{2}}^{I}} l_{\frac{N+M-1}{2}}=0$, exactly as for the corresponding passage used in terms of the moments.)

By using Equations $(10)_{1}$ and $(15)_{1}$, we find:

$$
\begin{aligned}
& \lambda_{A} F^{A}=\sum_{n=0}^{N} \lambda_{i_{1} \cdots i_{n}} \sum_{s=0}^{n} S_{j_{1} \cdots j_{s}}^{i_{1} \cdots i_{n}}(\vec{u}) F^{I j_{1} \cdots j_{s}}+ \\
& +\sum_{R=0}^{M} \lambda_{i_{1} \cdots i_{R}}^{R}\left[\sum_{s=0}^{N} Y_{j_{1} \cdots j_{s}}^{i_{1} \cdots i_{R}}(\vec{u}) F^{I j_{1} \cdots j_{s}}+\sum_{S=R}^{M} X_{j_{1} \cdots j_{S}}^{i_{1} \cdots i_{R}}(\vec{u}) F_{S}^{I j_{1} \cdots j_{S}}\right]
\end{aligned}
$$

It follows that the coefficient of $F^{I}$ is:

$$
\sum_{n=0}^{N} \lambda_{i_{1} \cdots i_{n}} S^{i_{1} \cdots i_{n}}(\vec{u})+\sum_{R=0}^{M} \lambda_{i_{1} \cdots i_{R}}^{R} Y^{i_{1} \cdots i_{R}}(\vec{u})
$$

and the coefficient of $F^{I j}$ is:

$$
\sum_{n=1}^{N} \lambda_{i_{1} \cdots i_{n}} S_{j}^{i_{1} \cdots i_{n}}(\vec{u})+\sum_{R=0}^{M} \lambda_{i_{1} \cdots i_{R}}^{R} Y_{j}^{i_{1} \cdots i_{R}}(\vec{u})
$$

where we have omitted the value for $n=0$, because, in the other summation, the index, $s$, goes from 0 to $n$; consequently, for $n=0$, there is not the value $s=1$.

By using Equations (11) and (14), the coefficient of $F^{I}$ becomes:

$$
\sum_{n=0}^{N} \lambda_{i_{1} \cdots i_{n}} u^{i_{1}} \cdots u^{i_{n}}+\sum_{R=0}^{M} \lambda_{i_{1} \cdots i_{R}}^{R} u^{i_{1}} \cdots u^{i_{R}}\left(u^{2}\right)^{\frac{N+M+1}{2}-R}
$$

while the coefficient of $F^{I j}$ becomes:

$$
\begin{aligned}
& \sum_{n=1}^{N} n \lambda_{i_{1} \cdots i_{n-1} j} u^{i_{1}} \cdots u^{i_{n-1}}+ \\
& +\sum_{R=0}^{M}\left[R \lambda_{j i_{2} \cdots i_{R}}^{R} u^{i_{2}} \cdots u^{i_{R}}\left(u^{2}\right)^{\frac{N+M+1}{2}-R}+(N+M+1-2 R) u_{j} \lambda_{i_{1} \cdots i_{R}}^{R} u^{i_{1}} \cdots u^{i_{R}}\left(u^{2}\right)^{\frac{N+M-1}{2}-R}\right]
\end{aligned}
$$

By calculating these expressions in $u_{i}=-\frac{1}{N+M+1} \frac{\lambda_{i}^{1}}{\lambda^{0}}$, we find $\tilde{\mu}$ and $\tilde{\mu}_{j}$, respectively, from which Equations (54) and (55) easily follow.

With the same passages, we find the other properties, that is, $\lambda$ appears in $\mu_{\tilde{B}}$ only for $\tilde{B}=0$ and $\lambda_{i}$ appears in $\mu_{\tilde{B}}$ only for $\tilde{B}=0$ or $\tilde{B}=j$. 
More precisely, $\mu_{\tilde{B}}$ is the coefficient of $F^{I \tilde{B}}$ in $\lambda_{A} F^{A}$, calculated in $u_{i}=-\frac{1}{N+M+1} \frac{\lambda_{i}^{1}}{\lambda^{0}}$; but, $\lambda$ appears in Equation (56) only for $n=0$, so that the other summation may assume only the value, $s=0$, giving only the coefficient of $F^{I}$ and not of the others, $F^{I \tilde{B}}$. Similarly, $\lambda_{i}$ appears in Equation (56) only for $n=1$, so that the other summation may assume only the value $s=0$ and $s=1$, giving only the coefficients of $F^{I}$ and $F^{I j}$ and not of the others, $F^{I \tilde{B}}$.

By using Equations (54) and (55), we can rewrite Equation (53) as:

$$
\frac{\partial h^{\prime k}}{\partial \tilde{\mu}}=-\frac{1}{N+M+1} \frac{\lambda_{k}^{1}}{\lambda^{0}} \frac{\partial h^{\prime}}{\partial \tilde{\mu}}+\frac{\partial h^{\prime}}{\partial \tilde{\mu}^{k}}
$$

which, by using Equations (50) and (52) becomes:

$$
\frac{\partial H^{\prime k}}{\partial \tilde{\mu}}=\frac{\partial H^{\prime}}{\partial \tilde{\mu}^{k}}
$$

This equation may be easily integrated; in fact, there exists surely a function, $\psi$, such that:

$$
H^{\prime}=\frac{\partial \psi}{\partial \tilde{\mu}}
$$

After that, Equation (58) says that:

$$
H^{\prime k}=\frac{\partial \psi}{\partial \tilde{\mu}^{k}}+\psi^{k}
$$

where $\psi^{k}$ is an arbitrary function that does not depend on $\tilde{\mu}$ and arises from the integration with respect to $\tilde{\mu}$.

\subsection{On the Eventual Condition $G^{k i}=G^{i k}$}

This symmetry condition $(2)_{2}$ has to be imposed only if there is a conservation of angular momentum, differently from Equation $(2)_{1}$, which holds surely, for physical reasons.

By using Equation (8), it becomes:

$$
\frac{\partial h^{\prime[k}}{\partial \lambda_{i]}}=0
$$

Now, thanks to Equations (54) and (55), we have:

$$
\frac{\partial h^{\prime k}}{\partial \lambda_{i}}=-\frac{1}{N+M+1} \frac{\partial h^{\prime k}}{\partial \tilde{\mu}} \frac{\lambda_{i}^{1}}{\lambda^{0}}+\frac{\partial h^{\prime k}}{\partial \tilde{\mu}_{i}}
$$

which, by using Equation (57) becomes:

$$
\frac{\partial h^{\prime k}}{\partial \lambda_{i}}=\frac{1}{(N+M+1)^{2}} \frac{\partial h^{\prime}}{\partial \tilde{\mu}} \frac{\lambda_{i}^{1} \lambda_{k}^{1}}{\left(\lambda^{0}\right)^{2}}-\frac{1}{N+M+1} \frac{\lambda_{i}^{1}}{\lambda^{0}} \frac{\partial h^{\prime}}{\partial \tilde{\mu}_{k}}+\frac{\partial h^{\prime k}}{\partial \tilde{\mu}_{i}}
$$

It follows that:

$$
\begin{aligned}
& \frac{\partial h^{\prime[k}}{\partial \lambda_{i]}}=-\frac{1}{N+M+1} \frac{1}{\lambda^{0}} \frac{\partial h^{\prime}}{\partial \tilde{\mu}_{[k}} \lambda_{i]}^{1}+\frac{\partial h^{\prime[k}}{\partial \tilde{\mu}_{i]}}= \\
& =\frac{1}{N+M+1} \frac{1}{\lambda^{0}} \frac{\partial h^{\prime}}{\partial \tilde{\mu}_{[i}} \lambda_{k]}^{1}+\frac{\partial h^{\prime[k}}{\partial \tilde{\mu}_{i]}}=\frac{\partial H^{\prime[k}}{\partial \tilde{\mu}_{i]}}
\end{aligned}
$$


where in the last passage, Equation (52) has been used.

Consequently, the condition (61) becomes:

$$
\frac{\partial H^{\prime[k}}{\partial \tilde{\mu}_{i]}}=0
$$

This equation, together with Equation (58), is nothing else than the integrability conditions, which allow one to obtain the scalar function, $\psi$, such that:

$$
H^{\prime}=\frac{\partial \psi}{\partial \tilde{\mu}} \quad, \quad H^{\prime k}=\frac{\partial \psi}{\partial \tilde{\mu}_{k}}
$$

Vice versa, from Equation (63), the conditions (58) and (62) easily follow. We may conclude that the four-potentials, $h^{\prime}$ and $h^{\prime k}$, are determined, except for an arbitrary scalar function, $\psi$, depending on $\lambda^{0}$ and $\mu_{\tilde{B}}$.

Let us now test the results of the present paper, by comparing their restriction to the 14-moments model with those found, for this particular case and in a completely different way, in $[5,6]$.

\section{The Particular Case with 14-Moments}

A comparison of the definition $(46)_{2}$ of $\mu_{\tilde{B}}$ with Equation $(28)_{2}$ shows that $\mu_{\tilde{B}}$ is equal to $\lambda_{\tilde{B}}^{I}$ calculated in $u_{i}=-\frac{1}{N+M+1} \frac{\lambda_{i}^{1}}{\lambda^{0}}$. Now, in Equation $(16)_{2}$ of [5], we find the expressions of $\lambda_{B}^{I}$ (which in that paper, were called $m^{B}$ ) in the 14-moments case; so, they allow one to obtain:

$$
\begin{aligned}
& \tilde{\mu}=\lambda-\frac{1}{4} \lambda_{i} \frac{\lambda_{i l l}}{\lambda_{p p q q}}+\frac{1}{16} \lambda_{i j} \frac{\lambda_{i l l} \lambda_{j l l}}{\left(\lambda_{p p q q}\right)^{2}}-\frac{3}{256}\left(\lambda_{p p q q}\right)^{-3}\left(\lambda_{i l l} \lambda_{i l l}\right)^{2} \\
& \tilde{\mu}_{i}=\lambda_{i}-\frac{1}{2} \lambda_{i j} \frac{\lambda_{j l l}}{\lambda_{p p q q}}+\frac{1}{8}\left(\lambda_{p p q q}\right)^{-2}\left(\lambda_{p l l} \lambda_{p l l}\right) \lambda_{i l l} \\
& \tilde{\mu}_{r s}=\lambda_{r s}-\frac{1}{8} \frac{\lambda_{i l l} \lambda_{i l l}}{\lambda_{p p q q}} \delta_{r s}-\frac{1}{4} \lambda_{r l l} \lambda_{s l l}\left(\lambda_{p p q q}\right)^{-1}
\end{aligned}
$$

where, in the present notation, $\lambda_{i l l}=\lambda_{i}^{1}$ and $\lambda_{\text {ppll }}=\lambda^{0}$.

Now, let us assume that $h^{\prime}$ depends on $\lambda_{A}$ as a composite function of $\lambda_{\text {ppll }}, \tilde{\mu}, \tilde{\mu}_{i}$ and $\tilde{\mu}_{r s}$ and of Equation (64); if we substitute it in $(4)_{1}$ of [5], we find that it is identically satisfied, as we expected for what was said above in the present paper.

Similarly, if we assume that:

$$
h^{\prime k}=-\frac{1}{4} \frac{\lambda^{k l l}}{\lambda_{p p l l}} h^{\prime}+H^{\prime k}\left(\lambda_{\text {ppll }}, \tilde{\mu}, \tilde{\mu}_{i}, \tilde{\mu}_{r s}\right)
$$

with $\tilde{\mu}, \tilde{\mu}_{i}$ and $\tilde{\mu}_{r s}$ given by Equation (64), we obtain that $(4)_{2}$ of [5] is identically satisfied, as we expected.

Regarding the expression of $H^{\prime k}$, we know from the representation theorems $[7,8,10]$ that

$$
H^{\prime k}=\psi_{1} \tilde{\mu}_{k}+\psi_{2} \tilde{\mu}_{k i} \tilde{\mu}_{i}+\psi_{3}\left(\tilde{\mu}^{2}\right)_{k i} \tilde{\mu}_{i}
$$

with $\psi_{1}, \psi_{2}$ and $\psi_{3}$ arbitrary scalar functions. Moreover, from the same representation theorems, we know that $H^{\prime}$ and $\psi_{i}$ are arbitrary functions of $\lambda_{p p q q}, \tilde{\mu}, \tilde{\mu}_{l l}, \tilde{\mu}_{l i} \tilde{\mu}_{l i}, \tilde{\mu}_{l i} \tilde{\mu}_{i j} \tilde{\mu}_{j l}, \tilde{\mu}_{i} \tilde{\mu}_{i}, \tilde{\mu}_{i} \tilde{\mu}_{i j} \tilde{\mu}_{j}$ and $\tilde{\mu}_{i} \tilde{\mu}_{i l} \tilde{\mu}_{l j} \tilde{\mu}_{j}$. 
Now we expect that there is a correspondence between the solution found in the present paper and that found in [5], with a completely different method. This is true; in fact, in [5] it has been found that $h^{\prime}$ is an arbitrary function of eight scalar variables $X_{1}-X_{8}$. By comparing their expressions (see Equation 6 of [5]), with the solution here found, we see that the correspondence between the scalars is the following one:

$$
\begin{aligned}
& \lambda^{0}=\lambda_{p p q q}=X_{1} \\
& \tilde{\mu}_{l l}=-\frac{5}{16} \frac{X_{2}}{X_{1}} \\
& \tilde{\mu}_{l i} \tilde{\mu}_{l i}=-\frac{1}{4} \frac{X_{3}}{X_{1}}+\frac{11}{256}\left(\frac{X_{2}}{X_{1}}\right)^{2} \\
& \tilde{\mu}_{l i} \tilde{\mu}_{i j} \tilde{\mu}_{j l}=-\frac{3}{8} \frac{X_{4}}{X_{1}}+\frac{3}{32} \frac{X_{3}}{X_{1}} \cdot \frac{X_{2}}{X_{1}}-\frac{29}{8^{4}}\left(\frac{X_{2}}{X_{1}}\right)^{3} \\
& \tilde{\mu}=\frac{1}{16} \frac{X_{5}}{X_{1}}+\frac{1}{32} \frac{X_{3}}{\left(X_{1}\right)^{2}}-\frac{3}{1024} \frac{\left(X_{2}\right)^{2}}{\left(X_{1}\right)^{3}} \\
& \tilde{\mu}_{i} \tilde{\mu}_{i}=\frac{1}{4} \frac{X_{6}}{X_{1}}-\frac{1}{32} \frac{X_{5} X_{2}}{\left(X_{1}\right)^{2}}+\frac{1}{8} \frac{X_{4}}{\left(X_{1}\right)^{2}}-\frac{1}{32} \frac{X_{3} X_{2}}{\left(X_{1}\right)^{3}}+\frac{1}{512} \frac{\left(X_{2}\right)^{3}}{\left(X_{1}\right)^{4}} \\
& \tilde{\mu}_{i} \tilde{\mu}_{i j} \tilde{\mu}_{j}=\frac{1}{4} \frac{X_{7}}{X_{1}}-\frac{3}{64} \frac{X_{2} X_{6}}{\left(X_{1}\right)^{2}}-\frac{1}{4}\left[\frac{1}{8} X_{3}-\frac{3}{128} \frac{\left(X_{2}\right)^{2}}{X_{1}}\right] \frac{X_{5}}{\left(X_{1}\right)^{2}}-\frac{5}{128} \frac{X_{2} X_{4}}{\left(X_{1}\right)^{3}}-\frac{1}{64} \frac{\left(X_{3}\right)^{2}}{\left(X_{1}\right)^{3}}+ \\
& +\frac{1}{128} \frac{X_{3}\left(X_{2}\right)^{2}}{\left(X_{1}\right)^{4}}-\frac{3}{2^{13}} \frac{\left(X_{2}\right)^{4}}{\left(X_{1}\right)^{5}} \\
& \tilde{\mu}_{i} \tilde{\mu}_{i l} \tilde{\mu}_{l j} \tilde{\mu}_{j}=\frac{1}{4} \frac{X_{8}}{X_{1}}-\frac{1}{16} \frac{X_{2} X_{7}}{\left(X_{1}\right)^{2}}+\frac{X_{6}}{X_{1}}\left[\frac{9}{16 \cdot 64}\left(\frac{X_{2}}{X_{1}}\right)^{2}-\frac{1}{32} \frac{X_{3}}{X_{1}}\right]+ \\
& +\frac{X_{5}}{X_{1}}\left\{\frac{3}{128} \frac{X_{2}}{X_{1}}\left[\frac{1}{2} \frac{X_{3}}{X_{1}}-\frac{3}{64}\left(\frac{X_{2}}{X_{1}}\right)^{2}\right]-\frac{1}{32} \frac{X_{4}}{X_{1}}\right\}+ \\
& +\frac{1}{32} \frac{X_{4}}{\left(X_{1}\right)^{2}}\left[-\frac{X_{3}}{X_{1}}+\frac{21}{64}\left(\frac{X_{2}}{X_{1}}\right)^{2}\right]+\frac{1}{128}\left(\frac{X_{3}}{X_{1}}\right)^{2} \frac{X_{2}}{\left(X_{1}\right)^{2}}+ \\
& -\frac{1}{64} \frac{15}{128} \frac{X_{3}}{\left(X_{1}\right)^{2}}\left(\frac{X_{2}}{X_{1}}\right)^{3}+\frac{9}{2^{17}} \frac{1}{X_{1}}\left(\frac{X_{2}}{X_{1}}\right)^{5}
\end{aligned}
$$

In order to verify these relations, it suffices to substitute Equation (64) in the lefthand sides and the expressions of $X_{1}-X_{8}$ (reported in Equation 7 of [5]) in the righthand sides; after that, we have simply to note that identities are obtained, thanks also to the Hamilton-Cayley theorem (see also on page 32 of [7]).

We note also that Equation (67) is invertible. In fact, the righthand side of Equation $(67)_{i}$ is a linear function in the variable $X_{i}$; moreover, the coefficient of $X_{i}$ and the term of zero degree in $X_{i}$ are functions, depending only on $X_{1}, X_{2}$ and $\cdots$ and $X_{i-1}$. This fact proves that Equation (67) is easily invertible.

For what concerns $h^{\prime k}$, let us analyze firstly the result of [5]. Equation 5 of this paper gives $h^{\prime k}$ and $h^{\prime}$ in terms of four arbitrary functions, $H_{0}, H_{1}, H_{2}$ and $H_{3}$, depending on the variables, $X_{1}-X_{8}$. By deducing from $(5)_{2}$ the function $H_{0}$ in terms of $h^{\prime}, H_{1}, H_{2}$ and $H_{3}$ and by substituting it in $(5)_{1}$, we find:

$$
h^{\prime k}=-\frac{1}{4} \frac{\lambda^{k l l}}{\lambda_{p p l l}} h^{\prime}+H_{1}\left(V_{1}^{k}-\frac{X_{2}}{8 X_{1}} V_{0}^{k}\right)+H_{2}\left(V_{2}^{k}-\frac{X_{3}}{8 X_{1}} V_{0}^{k}\right)+H_{3}\left(V_{3}^{k}-\frac{X_{4}}{8 X_{1}} V_{0}^{k}\right)
$$


where we have used Equation $(6)_{1}$ of [5].

After that, by using also Equations 6 and 7 of [5] and the Equation (64) of the present paper, we find the following identities:

$$
\begin{aligned}
V_{1}^{k}-\frac{X_{2}}{8 X_{1}} V_{0}^{k} & =4 X_{1} \tilde{\mu}^{k} \\
V_{2}^{k}-\frac{X_{3}}{8 X_{1}} V_{0}^{k} & =4 X_{1} \tilde{\mu}^{k i} \tilde{\mu}_{i}+\frac{3}{4} X_{2} \tilde{\mu}^{k} \\
V_{3}^{k}-\frac{X_{4}}{8 X_{1}} V_{0}^{k} & =4 X_{1} \tilde{\mu}^{k l} \tilde{\mu}^{l i} \tilde{\mu}_{i}+X_{2} \tilde{\mu}^{k i} \tilde{\mu}_{i}+\left[\frac{1}{2} X_{3}+\frac{3}{64}\left(X_{2}\right)^{2}\right] \tilde{\mu}^{k}
\end{aligned}
$$

by substituting these values in Equation (68), we find:

$$
h^{\prime k}+\frac{1}{4} \frac{\lambda^{k l l}}{\lambda_{\text {ppll }}} h^{\prime}=\psi_{1} \tilde{\mu}_{k}+\psi_{2} \tilde{\mu}_{k i} \tilde{\mu}_{i}+\psi_{3} \tilde{\mu}^{k l} \tilde{\mu}^{l i} \tilde{\mu}_{i}
$$

with

$$
\begin{aligned}
& \psi_{1}=4 X_{1} H_{1}+\frac{3}{4} X_{2} H_{2}+\left[\frac{1}{2} X_{3}+\frac{3}{64}\left(X_{2}\right)^{2}\right] H_{3} \\
& \psi_{2}=4 X_{1} H_{2}+X_{2} H_{3} \\
& \psi_{3}=4 X_{1} H_{3}
\end{aligned}
$$

From the arbitrariness of $H_{1}, H_{2}$ and $H_{3}$, that of $\psi_{1}, \psi_{2}$ and $\psi_{3}$ follows, and vice versa.

We note now that Equation (70) is equivalent to Equations (65) and (66) of the present article. So, we have found a complete proof that as the result of the present work, restricted to the 14 moments case, is the same of that known in the literature and found with a completely different method.

\section{Conclusions}

We consider very interesting the results here obtained, because until now, nobody has found exact solutions in Extended Thermodynamics with an arbitrary number of moments. This allows one to review the procedures previously used and which were based on Taylor's expansions. Moreover, it opens up the possibility for many other further deepening considerations; for example, one could try to extend this procedure to the case with additional symmetry conditions. We hope that this will be a great spur also for other researchers.

\section{Acknowledgments}

We thank two anonymous referees whose suggestions helped in the presentation of this article. One of the authors, M.C. Carrisi, thanks also the financial support to this research, through an "assegno di ricerca", by the University of Cagliari and the Fondazione del Banco di Sardegna, as a joint financing sponsor.

\section{References}

1. Carrisi, M.C. A further condition in the extended macroscopic approach to relativistic gases. Int. J. Pure Appl. Math. 2011, 67, 291-325. 
2. Carrisi, M.C. A generalized kinetic approach for the study of relativistic electron beams. Acta Appl. Math. 2012, 122, 107-116.

3. Kremer, G.M. Extended thermodynamics of molecular ideal gases. Contin. Mech. Thermodyn. 1989, 1, 21-45.

4. Kremer, G.M.; Beevers, C. Extended Thermodynamics of Dense Gases. In Recent Developments in Nonequilibrium Thermodynamics (Barcelona, 1983), Lecture Notes in Phys; Springer: Berlin, Germany, 1983; Volume 199, pp. 429-436.

5. Carrisi, M.C.; Mele, M.A.; Pennisi, S. An Extended model for dense gases and macromolecular fluids, obtained without using Taylor' s expansions. Int. J. Pure Appl. Math. 2009, 57, 27-55.

6. Carrisi, M.C.; Mele, M.A.; Pennisi, S. On Some Remarkable Properties of an Extended model for dense gases and macromolecular fluids. Proc. R. Soc. A. 2010, 466, 1645-1666.

7. Wang, C.C. On representations for isotropic functions. Arch. Ratl. Mech. Anal. 1969, 33, 249-287.

8. Smith, G.F. On isotropic functions of symmetric tensor, skew symmetric tensor and vectors. Int. J. Eng. Sci. 1971, 9, 899-916.

9. Pennisi, S. Some representation theorems in a 4-dimensional inner product space. Suppl. BUMI Fisica Mat. 1986, 5, 191-214.

10. Pennisi, S.; Trovato, M. On the Irreducibility of Professor G.F. Smith's Representations for isotropic functions. Int. J. Eng. Sci. 1987, 25, 1059-1065.

11. Pennisi, S.; Trovato, M. Mathematical characterization of functions underlying the principle of Relativity. Le Matematiche. 1989, XLIV, 173-203.

12. Liu, I.-S.; Müller, I. Extended thermodynamics of classical and degenerate ideal gases. Arch. Rat. Mech. Anal. 1983, 83, 285-332.

13. Liu, I.-S.; Müller, I.; Ruggeri, T. Relativistic thermodynamics of gases. Ann. Phys. 1986, 169, 191-219.

14. Müller, I.; Ruggeri, T. Rational Extended Thermodynamics. In Springer Tracts in Natural Philosophy, 2nd ed.; Springer-Verlag: New York, NY, USA, 1998; Volume 37.

15. Struchtrup, H. The BGK-model with velocity-dependent collision frequency. Cont. Mech. Thermodyn. 1997, 9, 23-31.

16. Struchtrup, H. On the number of moments in radiative transfer problems. Ann. Phys. 1998, $266,1-26$.

17. Struchtrup, H. Extended moment method for electrons in semiconductors. Physica A 2002, 275, 229-255.

18. Torrilhon, M.; Au, J.D.; Struchtrup, H. Explicit fluxes and productions for large systems of the moment method base on extended thermodynamics. Cont. Mech. Thermodyn. 2002, 15, 97-111.

19. Struchtrup, H. Heat transfer in the transition regime: Solution of boundary value problems for Grad's moment equations via kinetic schemes. Phys. Rev. E. 2002, doi: 10.1103/PhysRevE.65.041204.

20. Struchtrup, H. Stable transport equations for rarefied gas at high order in the Knudsen number. Phys. Fluids. 2004, 16, 3921-3934.

21. Struchtrup, H. Derivation of 13 moment equations for rarefied gas flow to second order accuracy for arbitrary interaction potentials. Multiscale Model. Simul. 2005, 3, 221-243. 
22. Borghero, F.; Demontis, F.; Pennisi, S. The Non-Relativistic Limit of Relativistic Extended Thermodynamics with Many Moments. Part I: The Balance Equations. In Proceedings of Wascom 2005; World Scientific: Singapore, 2005; pp. 47-52.

23. Carrisi, M.C.; Demontis, F.; Pennisi, S. The Non-Relativistic Limit of Relativistic Extended Thermodynamics with Many Moments. Part II: How it Includes the Mass, Momentum and Energy Conservation. In Proceedings of Wascom 2005; World Scientific: Singapore, 2005; pp. 95-100.

24. Dreyer, W.; Weiss, W. The Classical Limit of Relativistic Extended Thermodynamics. Ann. de l' Institut Henri Poincaré. 1986, 45, 401-418.

25. Carrisi, M.C.; Pennisi, S. Some Open Problems in non-linear Extended Thermodynamics and their Possible Solution. Ricerche Mat. 2011, 60, 45-56.

26. Enskog, D. K. Svenska Vetenscaps Akad. Handl. 1921, 63, No.4 and the English version by Brush, S. Kinetic Theory; Pergamon Press: Oxford, UK, 1972; Volume 3, p. 226.

27. Chapman, S.; Cowling, T. Mathematical Theory of Non-Uniform Gases; 3rd, Ed; Cambridge University Press: Cambridge, UK, 1970; pp. 1-448.

28. Kremer, G.M.; Rosa, E., Jr. On Enskog's dense gas theory. I. The method of moments for monoatomic gases. J. Chem. Phys. 1988, 89, 3240-3247.

29. Marques, W., Jr.; Kremer, G.M. On Enskog's dense gas theory. II. The linearized burnett equations for monatomic gases. Rev. Bras. Fis. 1991, 21, 402-417.

30. Ugawa, H.; Cordero, P. Extended Hydrodynamics from Enskog's equation for a two-dimensional system general formalism. J. Stat. Phys. 2007, 127, 339-358.

31. Arima, T.; Taniguchi, S.; Ruggeri, T.; Sugiyama, M. Extended Thermodynamics of dense gases. Cont. Mech. Thermodyn. 2012, 24, 271-292.

32. Liu, I.-S. Method of Lagrange multipliers for exploitation of the entropy principle. Arch. Ration. Mech. Anal. 1972, 46, 131-148.

33. Ruggeri, T.; Strumia, A. Main field and convex covariant density for quasi-linear hyperbolic systems. Relativistic Fluid Dyn. Ann. Ist. H. Poincaré. 1981, 34, 65-84.

34. Ruggeri, T. Galilean invariance and entropy principle for systems of balance laws. The structure of the extended thermodynamics. Cont. Mech. Thermodyn. 1989, 1, 3-20.

35. Liu, I.-S. On the structure of balance equations and extended field theories of mechanics. IL NUOVO CIMENTO 1986, 92B, 121-141.

36. Montisci, S.; Pennisi, S. Some useful Tensorial Identities for Extended Thermodynamics. Int. Electron. J. Pure Appl. Math. 2013, To be published

37. Pennisi, S.; Ruggeri, T. A New Method to Exploit the Entropy Principle and Galilean Invariance in the Macroscopic Approach of Extended Thermodynamics. In Ricerche di Matematica; Springer: Berlin/Heidelberg, Germany, 2006; Volume 55, pp. 319-339.

38. Carrisi, M.C.; Pennisi, S. The galilean relativity principle for a new kind of systems of balance equations in Extended Thermodynamics. Int. J. Pure Appl. Math. 2008, 42, 451-457.

39. Pennisi, S.; Scanu, A. Judicious Interpretation of the Conditions Present in Extended Thermodynamics. In Proceedings of Wascom 2003; World Scientific: Singapore, 2003; pp. 393-399. 
40. Pennisi, S.; Carrisi, M.C. On the Exact Macroscopic Approach to Extended Thermodynamics with 20 Moments. In Proceedings of Wascom 2003; World Scientific: Singapore, 2003; pp. 386-392.

(C) 2013 by the authors; licensee MDPI, Basel, Switzerland. This article is an open access article distributed under the terms and conditions of the Creative Commons Attribution license (http://creativecommons.org/licenses/by/3.0/). 